\title{
Mitotic Activity of Tympanic Membrane and External Auditory Canal Skin in Normal Mongolian Gerbil
}

\author{
Keehyun Park, MD, Young-Myoung Chun, MD, Seong-Mo Koo, MD and Seong-Kyun Kim, MD \\ Department of Otolaryngology, School of Medicine, Ajou University, Suwon, Korea
}

Korean Journal of Otolaryngology-Head and Neck Surgery 1997;40(7):976-83.

The following original article from Korean Journal of Otolaryngology-Head and Neck Surgery, "Mitotic Activity of Tympanic Membrane and External Auditory Canal Skin in Normal Mongolian Gerbil” by Keehyun Park, published on July 1997 has been withdrawn from publication by agreement among the authors, the journal Editor, Sung Won Chae, and the publisher ML communications Co., Ltd. The retraction has been agreed due to substantial overlap of the content of this article with previously published one. The author and the Journal apologize to readers.

Sung Won Chae, MD, PhD

Editor

Korean Journal of Otorhinolaryngology-Head and Neck Surgery 